\title{
A smart management system of electric vehicles charging plans on the highway charging stations
}

\author{
Ibrahim El-Fedany ${ }^{1}$, Driss Kiouach ${ }^{2}$, Rachid Alaoui ${ }^{3}$ \\ ${ }^{1,2}$ LPAIS Laboratory, Faculty of Sciences Dhar El Mahraz Sidi Mohamed Ben Abdellah University, Fez, Morocco \\ ${ }^{3}$ LRIT Laboratory, Faculty of Sciences, Mohammed V University, Rabat, Morocco \\ ${ }^{3}$ LASTIMI Laboratory, Higher School of Technology-Sales, Mohammed V University, Rabat, Morocco
}

\begin{tabular}{l} 
Article Info \\
\hline Article history: \\
Received Jan 11, 2021 \\
Revised Jun 24, 2021 \\
Accepted Jun 29, 2021 \\
\hline
\end{tabular}

\section{Keywords:}

Communication system

Electric car

Highway

Queuing time

Smart scheduling algorithm

\begin{abstract}
Electric vehicles (EVs) are seen as one of the principal pillars of smart transportation to relieve the airborne pollution induced by fossil $\mathrm{CO}_{2}$ emissions. However, the battery limit, especially where the journey is with a long-distance road remains the most formidable obstacle to the large-scale use of EVs. To overcome the issue of prolonged waiting charging time due to the large number of EVs may have a charging plan at the same charging station (CS) along the highway, we propose a communication system to manage the EVs charging demands. The architecture system contains a smart scheduling algorithm to minimize trip time including waiting time, previous reservations, and energy are needed to reach the destination. Moreover, an automatic mechanism for updating reservation is integrated to adjust the EVs charging plans. The results of the evaluation under the Moroccan highway scenario connecting Rabat and Agadir show the effectiveness of our proposal system.
\end{abstract}

This is an open access article under the CC BY-SA license.

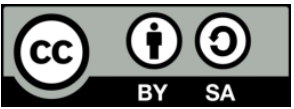

\section{Corresponding Author:}

Ibrahim El-Fedany

LPAIS Laboratory

Sidi Mohamed Ben Abdellah University

Dhar El Mahraz, Fès, Morocco

Email: ibrahim.elfedany@usmba.ac.ma

\section{INTRODUCTION}

Undesirable emissions of $\mathrm{CO}_{2}$ gases from the use of fossil fuel sources have now reached a menacing level that needs an immediate action of prevention by implementing eco-friendly policy [1]-[2]. Therefore, many countries, local government, and agency researchers in the world consider electric vehicles as a promising and suitable solution to resolve problems attached to transport sector and combat environmental pollution challenges [3]-[5], e.g. Electric vehicles (EVs) could possibly provide 45\% reduction in carbon emissions compared to traditional vehicles after taking into account the energy cost of production and usage [6].

Despite the promising signs, a few challenges remain with regard to the large spread of EVs, such as EV owners do not have the ability to charge their EVs at home, but must be based on public charging stations (CSs), longer time to charge [7], and the limited capacity of the battery that may not exceed 480 kilometers [8]. Meanwhile, electric vehicle drivers are always concerned with the charging problem due to the fewer charging station, and the decision of which charging station to choose [9]. Therefore, EV charging may take place several times during the journey when the trip is with long distance. In this context, the management of EV charging demands, especially on long-distance trip, is necessary to success and long-term widespread of EV. 
Most studies investigate charging scheduling for the use case about where and which CS to charge EVs in urban environments, a shorter travel distance between EVs and CSs. Ji et al. [10], propose a strategy to alleviate the unbalanced queuing problem of CSs at the dynamic peak shift of charging demand. A distributed architecture to suggest a set of the nearest charging stations for EV recharging using a distributed ant system algorithm [11]. Zhang, et al. [12], authors propose a formalization of the EV charging scheduling problem as a markov decision process and deep reinforcement learning algorithms to reduce the EVs total charging time. To minimize the total elapsed time by considering the traffic condition, the remaining energy in the battery, and the CS queuing length, the EV charging scheduling method is proposed in [13]. El-Fedany et al. [14], we propose an application design allowing the management of EVs charging demands, the CS selected decision based on minimizing the trip duration including waiting and charging time. Moreover, we proposed a system design update dynamically the EVs charging plans on real time [15]. A strategy to assign each EV to the most suitable CS according to a method that will minimize the waiting time [16]. The traffic information and map information are combined to obtain path network model data and CS queuing times that is used to solve the EV path planning problem [17]. the optimal route through a CS selected proposed in [18], the selection decision is making by the proposed algorithm, which combine reinforcement learning and neural networks for minimizing EVs trip time. Eventually, we have proposed in [19] a smart architecture system to manage EV charging plans and fixed and mobile charging stations, the system proposed is composed of a set of proposed algorithms and the recommendation of an appropriate CS for an EV charging request based on minimizing the total trip time including charging and waiting time at CS.

The EV charging-time limitation in highway environments is not as densely researched as that of urban environments. A few previous works have addressed CS selection decision to minimize the EVs charging trip duration in highway, by monitoring the CSs status. The others in [20] propose a scheduling method for EVs charging plans on a highway trip based on the A* search algorithm, an extension of Edsger Dijkstra's algorithm, to reduce the travel times, the decision is applied at EV level with CS parameters. The work in [21] presents a negotiation strategy to coordinate the queues among the CSs along a highway, by means of the vehicle-to-vehicle and vehicle-to-infrastructure communications, and a distributed algorithm is presented to schedule EVs so that each EV makes a suitable decision to avoid long waiting times. A study presented in [22] that aims to develop an algorithm of optimizing recharging stops along ontario's highway and examines the difference in time between EVs and standard vehicles. Chen et al. [23] propose a strategy for providing synchronous charging services for EVs to reduce the queue length at the highway CS.

As EVs get on the highway and when their Batteries reach a certain threshold, they are sending a charging reservation request to GA who is responsible for generating and updating charging plans. Unlike other approaches, we focus on trip time not only waiting time, and the decision making by GA takes into account the CS status in real-time to recommend and update the charging plan.

Our contribution is summarized as follows:

- We propose a system design to manage the charging reservation requests and avoid imbalance loading energy due to poor distribution of EVs on the CSs along a highway.

- We propose an architecture algorithm to manage the EVs charging plans and recommend a suitable CS where the trip time is the minimum including waiting and charging time. Furthermore, GA automatically updates the charging plan of EVs on-the-move on the highway when CSs conditions are changed by new reservations of EVs incoming.

- We developed a simulation framework and implemented our approach in a use case for Moroccan highway traffic connecting Rabat and Agadir, that accounts CSs and EVs specific characteristics and variable highway speed limitations.

The rest of this paper is organized as follows. Section 2 presents the architecture and the operating of the proposed system including system entity definition and assumption. In section 3, we present the detail of the proposed system algorithms. The evaluation of the effectiveness of proposed system algorithms is presented in section 4 . Finally, section 5 contains the conclusion of this work.

\section{A SYSTEM PROPOSED}

\subsection{Assumptions and system architecture}

The Figure 1 illustrates the global deseign of the system proposed which is composed of three main elements such as: charging stations, electric vehicles, and global aggregator genetic algorithm (GA). A detailed description of each element is given as follow:

- Electric vehicle: the work treats a scenario where electric vehicles are moving on the long distance (highway) and periodiclly look at the battery level. When the EV user enters in highway than he wants making a charging reservation or the current battery level is below a certain threshold, the EV driver 
sends a charging request into the GA in order to select a suitable CS by taking into account the information about EV's battery level and current location and the exit point of highway that leads to its final destination.

- Charging station: they are generally taken place at different locations on the highway, and each CS contains a set of parking spaces equipped with charging sockets. Thus, all CSs, based on Algorithm 1, send their status such as queuing time, number of charging slots into GA, and then the GA is able to estimate the charging and waiting time at each CS.

- Global aggregator: The GA is a central system to serve and cooperate all system entities (CSs and EVs) using cellular communication networks, and furthermore the GA center allows the selection of the appropriate $\mathrm{CS}$ for the EVs charging requests, this decision based on the information sent by the EVs and CSs in real time. The main algorithm set up on GA to make the CS selection decision is Algorithm 2 that is detailed in section 3.2 .

In this paper, we assume that all EVs are equipped with a global position system GPS [24], [25] that contains their own movement information, including current location and speed, and We also assume a seamless signaling exchange between CS, EVs, and GA across a reliable channel, such as 3G/LTE. Therefore, the EV needs charging reservation can be informed by the GA with charging planning. Likewise, all CSs apply first-come, first-served (FCFS) policy, the first EV that comes to CS is the first that is served, to manage the EVs at its parking lot, thereby when all CS sockets are occupied, the incoming EVs have to wait until one socket becomes available. Furthermore, we consider an unlimited number of places for parking at each CS.

\subsection{System operation}

follows:

A typical communication system among EVs and GA center is shown in Figure 2, and described as

- Phase 1: EV gets the highway and request the GA for a charging reservation, the request contains some EV information such as the current position, the current SoC, and the location of the exit point where the EV gets off the highway.

- Phase 2: The GA server permanently receives informations from CSs (current queuing time, and number of waiting vehicles), based on Algorithm 2, the GA selects the optimal CS for EV that have sent the charging request being processed, then informs all the EVs having an updating of the charging plan due to the new reservation.

- Phase 3: When an EV get in the parking area of its recommended CS, then this latter sends a message contains its current state to GA, then the EV reservation is canceled and GA server saves the last changing informations that will be used for the next charging demands.

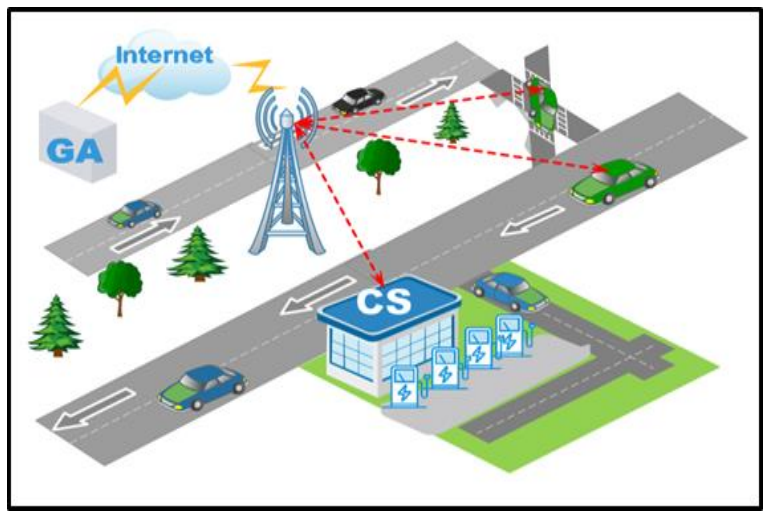

Figure 1. System architecture

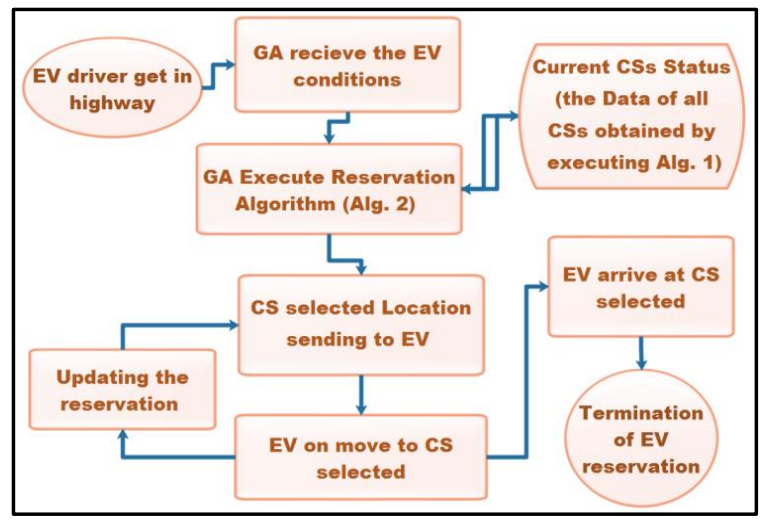

Figure 2. System cycle flowchart

\section{THE PROPOSED ALGORITHM AND RESEARCH METHOD}

Our proposed system algorithm is composed of two algorithms, the first is responsible for calculating the queuing time at CS according to the EVs parked in it, and the second is taking charge of making a decision of the optimal CS selection to each EV requesting charging reservation. The variables used in the system algorithms are described in Table 1. 
Table 1. List of nomenclature

\begin{tabular}{|c|c|}
\hline Symbol & Description \\
\hline TSlot & Output of algorithm1 containing available queuing time per each socket at charging station \\
\hline $\mathrm{N}_{\text {slot }}$ & The number of charging soket set up at charging station \\
\hline$T^{\text {cur }}$ & Current local time \\
\hline $\mathrm{N}_{\mathrm{ev}}$ & The number of EV parket at CS \\
\hline$\beta_{j} / \beta$ & charging rate of electricity power in $\mathrm{CS}_{\mathrm{j}} / \mathrm{CS}$ \\
\hline $\mathrm{E}_{\mathrm{ev}_{\mathrm{j}}}^{\max }$ & Maximum capacity of $\mathrm{EV}_{\mathrm{j}}$ battery \\
\hline $\mathrm{E}_{\mathrm{ev}_{j}}^{\mathrm{cur}}$ & Current charging level of $\mathrm{EV}_{\mathrm{j}}$ battery \\
\hline $\mathrm{T}_{\mathrm{ev}_{\mathrm{j}}}^{\mathrm{fin}}$ & Estimated time to finish the charging $\mathrm{EV}_{\mathrm{j}}$ battery \\
\hline TR & Form of dictionary list to save EVs reservation, pair of EV and their $\mathrm{CS}$ recommended \\
\hline TCR & Temporary list similarly to TR list, a clone of TR list \\
\hline MTSlot & List containing the TSlot lists of all CSs \\
\hline $\mathrm{T}^{\text {minChar }}$ & Variable used save the minimum trip duration \\
\hline $\mathrm{CS}_{\mathrm{j}_{\min }}$ & Variable used save the CS recommended \\
\hline $\mathrm{N}_{\mathrm{CSi}}$ & Numbers of CSs, where $E V_{i}$ can get a charging reservation \\
\hline $\mathrm{T}_{\mathrm{i}, \mathrm{j}}^{\mathrm{arr}}$ & The arrival time of $\mathrm{EV}_{\mathrm{i}}$ to $\mathrm{CS}_{\mathrm{j}}$. \\
\hline $\mathrm{T}_{\mathrm{i}, \mathrm{j}}^{\mathrm{tr} \text { tran }}$ & The travelling time between $\mathrm{EV}_{\mathrm{i}}$ location and $\mathrm{CS}_{\mathrm{j}}$. \\
\hline $\mathrm{T}_{\mathrm{i}, \mathrm{j}}^{\mathrm{ch}}$ & Estimated charging time of $\mathrm{EV}_{\mathrm{i}}$ \\
\hline $\mathrm{E}_{\mathrm{i}, \mathrm{j}}^{\mathrm{tran}}$ & Energy consumed for traveling $E V_{i}$ toward $\mathrm{CS}_{\mathrm{j}}$ \\
\hline SEVi & $\mathrm{EV}_{\mathrm{i}}$ Moving speed \\
\hline$\alpha$ & Rate of energy consumed per meter \\
\hline
\end{tabular}

\subsection{CS queuing time}

We assume in Algorithm 1 that each charging station spread on the highway has a number of charging sockets, denoted Ns, and adopts via several locations parallel charging processes, therefore, after the distribution, according to FCFS policy, of electric vehicles parked in CS to charge, the minimum value calculated for one of the CS charging slots become the waiting time in CS. The distribution operates as follows: At first, we suppose that each slot is represented under an index on the list Tslot, the size of this latter is denoted by $N_{\text {slot }}$, and initialized by $\mathrm{T}^{\text {cur }}$, line 1 to 3 .

The FCFS policy is applied to distribute the EVs parked in CS for charging, line 5, and each EV is attributed to a charging slot, line 6 to 10 Within each iteration, the EV charging time will be calculated, obtained by $\frac{E_{e v_{j}}^{\max }-E_{e v_{j}}^{c u r}}{\beta}$, and added to TSlot.get(0), the first available time at the list top, line 7 to line 8 . And then, TSlot list will be sorted by ascending order, these operations are repeated until all EVs have been processed. Finally, the algorithm returns TSlot list, line 12.

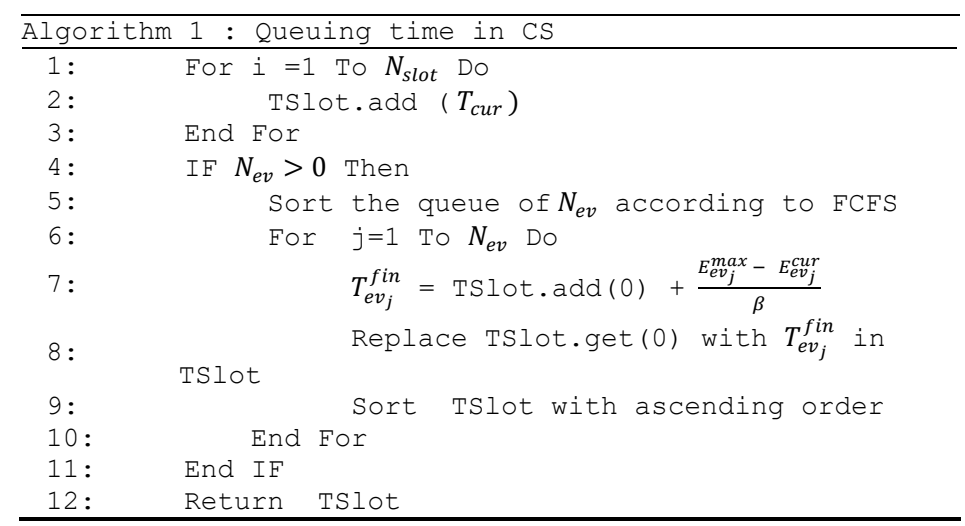

\subsection{Detail of CS selection algorithm}

The essential task of Algorithm 2 is to select the optimal CS for a reservation request of an $\mathrm{EV}_{\mathrm{r}}$, at the same time if this selection has an impact on the previous reservation, due to the arrival of $\mathrm{EV}_{\mathrm{r}}$ before the electric vehicles that had made the previous charging reservations in the same recommended CS, the algorithm sends a change notification of charging plan into the EVs having the change.

The CS recommendation based on the minimization of the trip duration expressed as follow:

$$
\begin{aligned}
& \mathrm{T}_{i, j}^{\text {Trip }}=\mathrm{T}_{\mathrm{i}, \mathrm{j}}^{\text {arr }}+\mathrm{T}_{\mathrm{i}, \mathrm{j}}^{\text {wait }}+\mathrm{T}_{\mathrm{i}, \mathrm{j}}^{\text {char }}+\mathrm{T}_{\mathrm{i}, \mathrm{j}, \mathrm{d}}^{\text {trans }} \\
& \mathrm{T}_{\mathrm{i}, \mathrm{j}}^{\text {arr }}=\mathrm{T}^{\text {curr }}+\mathrm{T}_{\mathrm{i}, \mathrm{j}}^{\text {trans }}
\end{aligned}
$$




$$
\begin{aligned}
& \mathrm{T}_{i, j}^{\text {char }}=\frac{E_{i}^{\text {max }}-E_{i}^{\text {curr }}+E_{i, j}^{\text {trans }}}{\beta_{j}} \\
& E_{i, j}^{\text {trans }}=\left(\operatorname{SEVix} \mathrm{T}_{i, j}^{\text {trans }} \times \alpha\right) \\
& \mathrm{T}_{i, j}^{\text {wait }}=\max \left[0, M T \operatorname{Mrlot}\left(\mathrm{CS}_{\mathrm{j}}\right) \cdot \operatorname{get}(0)-\mathrm{T}_{\mathrm{i}, \mathrm{j}}^{\mathrm{arr}}\right]
\end{aligned}
$$

here, the waiting time in $\mathrm{CS}_{\mathrm{j}}$ denotet by $\operatorname{MTs} \operatorname{sot}\left(\mathrm{CS}_{\mathrm{j}}\right)$.get $(0)$ and it is calculated by $\mathrm{GA}$ in view of all previous EVs charging reservations.

To achieve the goal, we use three variables list, TR where the reservations are saved, MTslot that contains all queues list of CSs and giving by Algorithm 1 line 2, and TCR list that is used at each new charging request by filling it with all reservations copy stored in TR list, line 3 . The loop, line 5 to line 18 , is responsible for assigning an optimal $\mathrm{CS}$ to each $\mathrm{EV}_{\mathrm{i}}$, i.e. the CS where the trip time is the minimum, by looking over all the CSs located on the same direction of $\mathrm{EV}_{\mathrm{i}}$, line 8 to 16, and taking into consideration the sufficient quantity of energy for arriving $\mathrm{EV}_{\mathrm{i}}$ to CSs, line 9. Then, the selection of the EV, which will arrive first at its recommendation, is obtained by sorting with ascending order of the TCR list according to $\mathrm{T}_{i, j}^{\mathrm{arr}}$, line 19. Thus, if the CS selected is different from what is in the TR reservation list, line 22, the charging CS is updated in line 23 and then the notification for changing the EV charging plan is sent in line 24. Therefore, EV is added to the reservation queuing of CS selected and removed from the TCR list, line 27 to line 28 . The previous operations are repeated until to serve all EV in the TCR list, loop of line 4 to line 29.

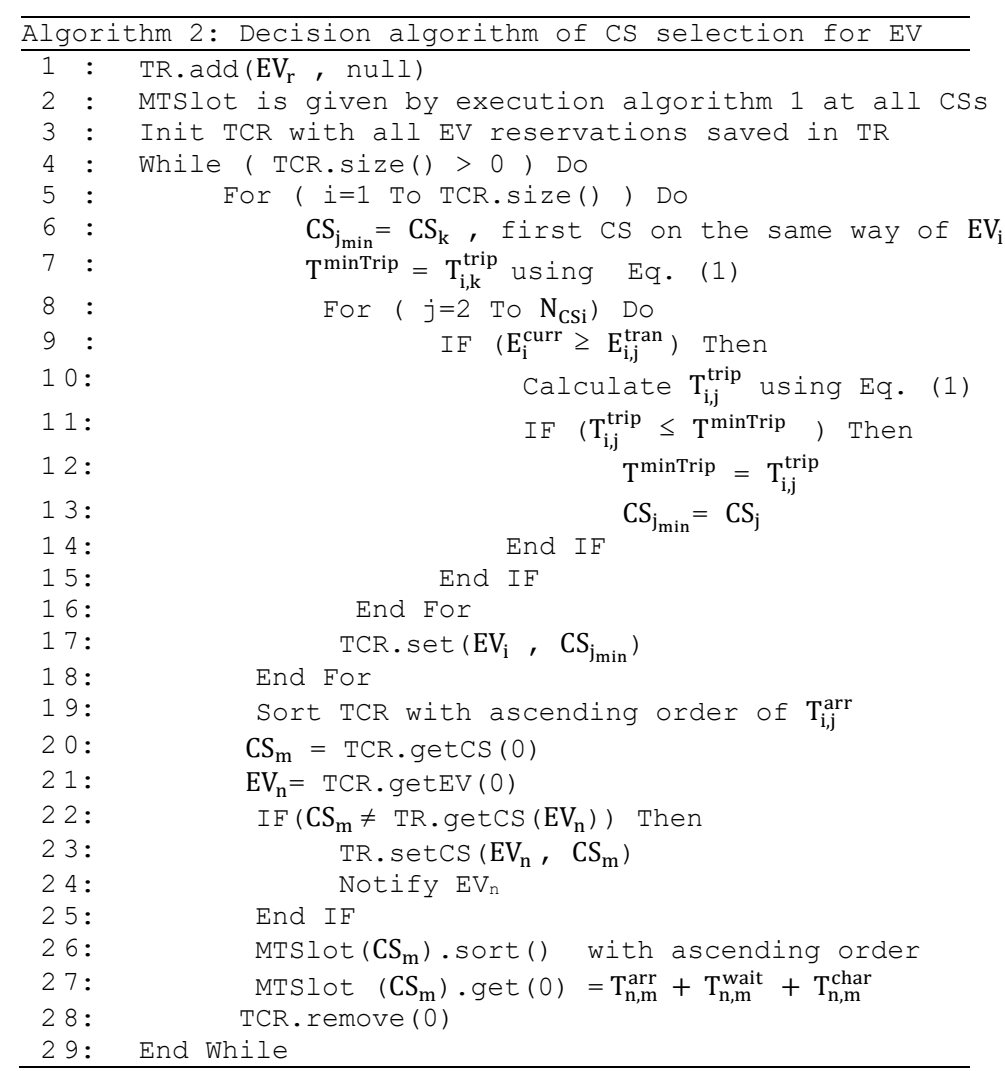

\section{A PERFORMANCE EVALUATION}

\subsection{Configurations of simulation system}

We present, in this section, the description in detail the system simulation' parameters setting. In fact, we built and developed in Java language a system simulation of discrete events to evaluate the proposed algorithms. Moreover, we presente, as a case study, a simulation of EVs network on the highway from Rabat to Agadir, Moroccan cities, about $540 \mathrm{~km}$. We obtained the locations of the charging stations in the highway from Google Map. As shown in Figure 3, we assume that we have a CS every $50 \mathrm{~km}$. Therefore, we get 10 fast charging stations. Each CS contains five charging slots, each of which has $80 \mathrm{~kW}$, the rate of the charge socket.

Similarly, we suppose that the simulation network path has 1,000 EVs of deferential cities located near the highway and can travel through it. Thus, each electric vehicle joining the system network of 
simulator is initialized in a uniform and normal distribution with a maximum charging parameter such as 100 $\mathrm{KWh}$ of the capacity maximum of battery, $10 \mathrm{KWh}$ per $100 \mathrm{~km}$ of the average consumption, $10 \%$ for the $\mathrm{SoC}$ and $120 \mathrm{Km}$ per hour of maximum speed. To evaluate the effectiveness of our proposed system algorithm, the simulation is performed in a variety of conditions, e.g. we consider normal and uniform distribution of different EVs charging demands, where they are generated randomly with varying numbers. Thus, the EVs energies and positions are updated every $0.1 \mathrm{~s}$ on the way or in a CS.

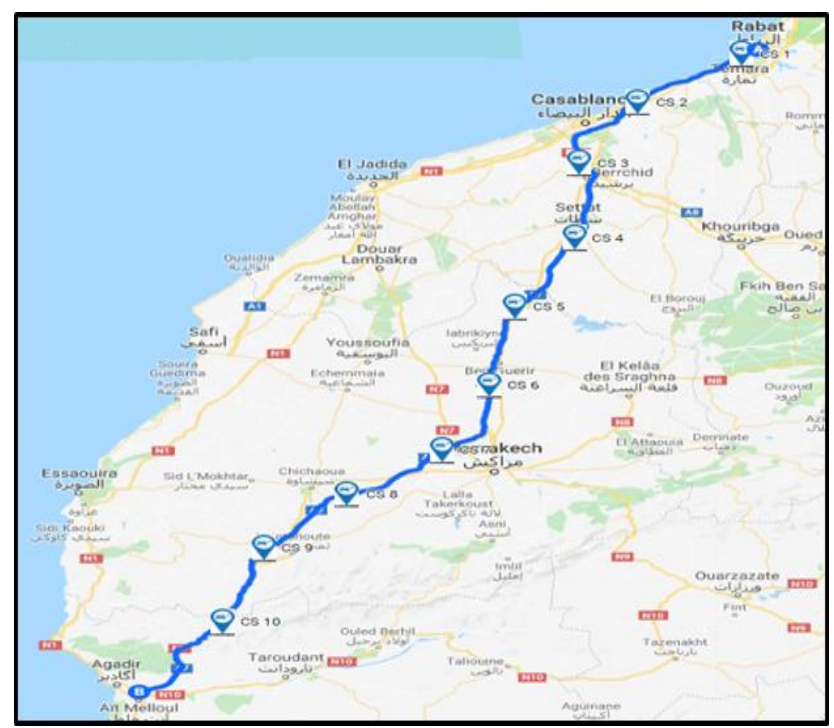

Figure 3. Simulation scenario of Moroccan highway Rabat-Agadir

\subsection{Results}

The proposed system strategy, based on the minimum trip time (MTT) and the updating regularly whether that currently selected CS is still the best choice, is evaluated and compared with the minimum waiting time (MWT) strategy, without reservation update like others previous works, to recommend the CS. We evaluate two performance metrics as follows: average trip times (ATT): The average time that an EV spent for its highway trip, through recharging service at an intermediate CS, and number of decision reservation changes (N-EV): The number of electric vehicles that have changed the charging plan along the trip time. In Figure 4, we present the average trip duration of EV charging demands on the basis of MTT and MWT strategies and with varied distributions of EV charging demands. We can see in this figure, result was obtained according to different numbers of EV charging demands, that the MTT curve is under WTT curve. Consequently, the better performance is offered by the proposed system with the lower average trip duration, as compared to MWT strategy, which can select the CS with minimum waiting time, but without updating the choice on each changing of CS status due to the nearest EVs incoming makes the first choice not been better. Moreover, our strategy will be able to more reduce the total trip time, especially when the number of EV charging demand increased, and even if increasing the number of charging slots at CSs Figure 5, the proposed system still shows their shorter charging trip time than MWT strategy.

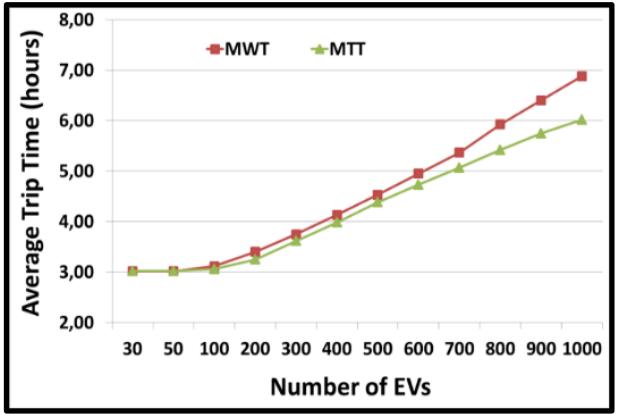

Figure 4. The influence of EVs number on trip time

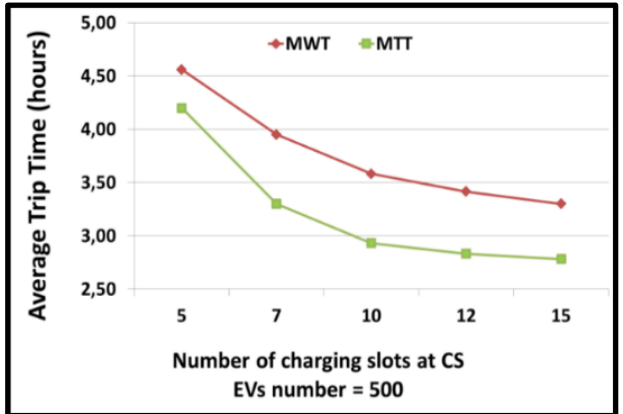

Figure 5. The influence of number charging slots on trip time 
In Figure 6, the number of reservations updating shows how important and performance of our system proposed, where an updated CS-selection is made at each changing CS conditions, such that the EV charging reservation would be adjusted depending on the new incoming EV charging reservation. Moreover, the updating of charging plan increases when the number EV charging demand increases, which explains the impact of our proposed system, especially when the number EV is very high.

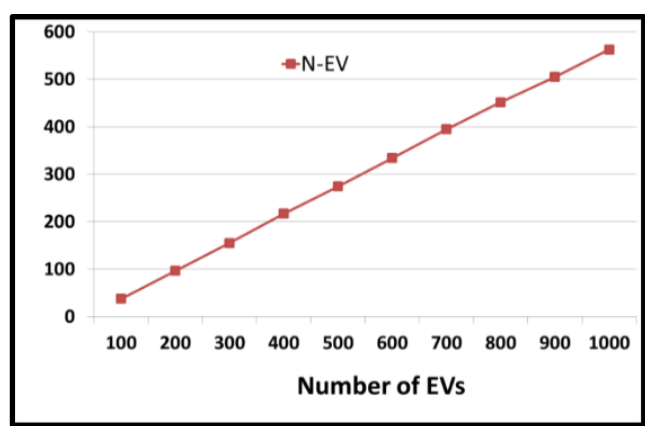

Figure 6. The number of EVs charging plans that have changed

\section{CONCLUSION}

In this paper, we addressed the problem of distributing electric vehicle flows in the highway transport network, by proposing a CS selection scheme in a charging management system in order to minimize the total EV trip time including charging and waiting time of EVs in the charging stations. The system composed of a global aggregator that is the responsible for making the CS selection decision and updating automatically the EV charging plans, especially when the status of the CS selected is changed by a new EVs incoming. Evaluation results, under highway scenario linking Rabat and Agadir cities in Morocco, showed the performance and effectiveness of our proposed system in terms of a shorter EVs trip time through intermediate charging station.

\section{REFERENCES}

[1] C. Haibo, et al., "Economic development and environmental sustainability-the case of foreign direct investment effect on environmental pollution in China," Environmental Science and Pollution Research, vol. 26, no. 7, pp. 7228-7242, 2019, doi: 10.1007/s11356-019-04167-5.

[2] H. P. Nguyen, et al., "Learned experiences from the policy and roadmap of advanced countries for the strategic orientation to electric vehicles: A case study in Vietnam," Energy Sources, Part A: Recovery, Utilization, and Environmental Effects, pp.1-10, 2020, doi: 10.1080/15567036.2020.1811432.

[3] C. Yang, et al., "Efficient energy management strategy for hybrid electric vehicles/plug-in hybrid electric vehicles: Review and recent advances under intelligent transportation system," IET Intelligent Transport Systems, vol. 14, no. 7, pp. 702-711, 2020, doi: 10.1049/iet-its.2019.0606.

[4] A. Ghosh, "Possibilities and challenges for the inclusion of the Electric Vehicle (EV) to reduce the carbon footprint in the transport sector: A review," Energies, vol. 13, no. 10, p. 2602, 2020, doi: 10.3390/en13102602.

[5] A. Muñoz-Villamizar, J. R. Montoya-Torres, and J. Faulin, "Impact of the use of electric vehicles in collaborative urban transport networks: A case study," Transportation Research Part D: Transport and Environment, vol. 50, pp. 40-54, 2017, doi: 10.1016/j.trd.2016.10.018.

[6] X. Zhang, et al., "Comparison of Climate Change Impact Between Power System of Electric Vehicles and Internal Combustion Engine Vehicles," Chinese Mate. Conf., Singapore, 2017, pp. 739-747, doi: 10.1007/978-981-13-0158-2_75.

[7] A. Gusrialdi, Z. Qu, and M. A. Simaan, "Distributed scheduling and cooperative control for charging of electric vehicles at highway service stations," IEEE Transactions on Intelligent Transportation Systems, vol. 18, no. 10, pp. 2713-2727, 2017, doi: 10.1109/TITS.2017.2661958

[8] A. E. Grunditz and T. Thiringer, "Performance analysis of current BEVs based on a comprehensive review of specifications," IEEE Transactions on Transportation Electrification, vol. 2, no. 3, pp. 270-289, 2016, doi: 10.1109/TTE.2016.2571783.

[9] F. Xie, et al., "Huang, Y. Long-term strategic planning of inter-city fast charging infrastructure for battery electric vehicles," Transportation Research Part E: Logistics and Transportation Review, vol. 109, pp. 261-276, 2018, doi: 10.1016/j.tre.2017.11.014.

[10] C. Ji, et al., "A Personalized Fast-Charging Navigation Strategy Based on Mutual Effect of Dynamic Queuing," IEEE Transactions on Industry Applications, vol. 56, no. 5, pp. 5729-5740, 2020, doi: 10.1109/TIA.2020.2985641. 
[11] M. Elgarej, M. Khalifa, and M.Youssfi, "Optimized path planning for electric vehicle routing and charging station navigation systems," International Journal of Applied Metaheuristic Computing (IJAMC), vol. 11, pp. 58-78, 2020, doi: 10.4018/978-1-7998-5339-8.ch094.

[12] C. Zhang, et al., "Effective Charging Planning Based on Deep Reinforcement Learning for Electric Vehicles," IEEE Transactions on Intelligent Transportation Systems, vol. 22, no. 1, pp. 542-554, 2020, doi: 10.1109/TITS.2020.3002271.

[13] L. P. Qian, et al., "Electric Vehicles Charging Scheduling Optimization for Total Elapsed Time Minimization," 2020 IEEE 91st Vehicular Technology Conference (VTC2020-Spring), Antwerp, Belgium, pp. 1-5, 2020, doi: 10.1109/VTC2020-Spring48590.2020.9128915.

[14] I. El-Fedany, D. Kiouach, and R. Alaoui, "Application Design Aiming to Minimize Drivers' Trip Duration through Intermediate Charging at Public Station Deployed in Smart Cities," World Electric Vehicle Journal, vol. 10, no. 4, p. 67, 2019, doi: 10.3390/wevj10040067.

[15] I. El-Fedany, D. Kiouach, and R. Alaoui. "System architecture to select the charging station by optimizing the travel time considering the destination of electric vehicle drivers in smart cities," Bulletin of Electrical Engineering and Informatics, vol. 9, no. 1, pp. 273-283, 2020, doi: 10.11591/eei.v9i1.1564.

[16] X. Bi, et al., "Distributing electric vehicles to the right charging queues," In 2019 IEEE International Symposium on Circuits and Systems (ISCAS), Sapporo, Japan, pp. 1-5, 2019, doi: 10.1109/ISCAS.2019.8702177.

[17] D. Ding, et al., "Electric Vehicle Charging Warning and Path Planning Method Based on Spark," IEEE Access, vol. 8, pp. 8543-8553, 2020, doi: 10.1109/ACCESS.2020.2964307.

[18] K. B. Lee, et al., "Deep Reinforcement Learning Based Optimal Route and Charging Station Selection," Energies, vol. 13, no. 23, pp. 6255, 2020, doi: 10.3390/en13236255.

[19] I. El-fedany, D. Kiouach, and R. Alaoui, "Rachid. A Smart Coordination System Integrates MCS to Minimize EV Trip Duration and Manage the EV Charging, Mainly at Peak Times," International Journal of Intelligent Transportation Systems Research, pp. 1-14, 2021, doi: 10.1007/s13177-021-00258-1.

[20] V. del Razo and H. A. Jacobsen, "Smart charging schedules for highway travel with electric vehicles," IEEE Transactions on Transportation Electrification, vol. 2, no. 2, pp. 160-173, 2016, doi: 10.1109/TTE.2016.2560524.

[21] A. Gusrialdi, Z. Qu, and M. A. Simaan, "Distributed scheduling and cooperative control for charging of electric vehicles at highway service stations," IEEE Transactions on Intelligent Transportation Systems, vol. 18, no. 10, pp. 2713-2727, 2017, doi: 10.1109/TITS.2017.2661958.

[22] A. Stabile, et al., "An Algorithm for Optimization of Recharging Stops: A Case Study of Electric Vehicle Charging Stations on Canadian's Ontario Highway 401," Energies, vol. 13, no. 8, p. 2055, 2020, doi: 10.3390/en13082055.

[23] L. Chen, et al., "Study of a new quick-charging strategy for electric vehicles in highway charging stations," Energies, vol. 9, no. 9, p. 744, 2016, doi: 10.3390/en9090744.

[24] B. Zhou, et al., "On the spatial error propagation characteristics of cooperative localization in wireless networks," IEEE Transactions on Vehicular Technology, vol. 66, no. 2, pp. 1647-1658, 2016, doi: 10.1109/TVT.2016.2555329.

[25] B. Zhou, Q. Chen, and P. Xiao, "The error propagation analysis of the received signal strength-based simultaneous localization and tracking in wireless sensor networks," IEEE Transactions on Information Theory, vol. 63, no. 6, pp. 3983-4007, 2017, doi: 10.1109/TIT.2017.2693180. 